\title{
DEGENERATIONS OF MONOMIAL IDEALS
}

\author{
HEATHER Russell
}

\begin{abstract}
We describe the degenerations of monomial ideals in $K[[x, y]]$ with $\operatorname{Aut}(K[[x, y]])$-orbit of dimension at most 3. In particular, we determine the monomial ideals that any power of $\left(x, y^{4}\right)$ can degenerate to and make a conjecture about all the ideals that the powers of $\left(x, y^{4}\right)$ can degenerate to. We also give some numerical evidence linking the characteristics in which one ideal degenerates to another with the enumeration of lattice paths.
\end{abstract}

\section{Introduction}

Let $I$ be an ideal of finite colength $n$ in the ring $K[[x, y]]$ for an arbitrary field $K$. Then $I /(x, y)^{n}$ corresponds to a point $p$ in the Grassmanian parametrizing codimension $n$ subspaces of $K[[x, y]] /(x, y)^{n}$. The automorphisms of $K[[x, y]]$ act on this Grassmanian. We will say that an ideal $J$ is a degeneration of $I$ if $J /(x, y)^{n}$ corresponds to a point in the closure of the orbit of $p$ under this action. The question of how an ideal can degenerate is a generalization of both the following two questions.

First, it is a generalization of a question about the stratification of local Hilbert schemes by Hilbert functions [12]. Each stratum can be decomposed into cells corresponding to ideals with given initial monomial ideal. The question is which strata are in the closure of others.

Second, the question of how ideals can degenerate is a generalization of the adjacency problem [1], the question of how a given type of singularity can degenerate. If the local equation of the singularity is contained in an ideal, then the local equation of any degeneration of the singularity is contained in a degeneration of that ideal.

In this paper, we answer the question of how the ideal $I$ can degenerate (modulo some computer programmable calculations for each individual ideal) in the case where $I$ is a monomial ideal with orbit of dimension 3 or less.

While our algorithmic answer still leaves a lot to be understood, we also study some examples where some order seems to emerge out of the chaos.

\section{Measuring Sequences}

Following the notation of [10] rather than the more general notation of [9], we will say that a monomial ideal $I$ in $K[[x, y]]$ has measuring sequence $\{a, b\}$ if

Received June 9, 2003.

2000 Mathematics Subject Classification. 14C05, 14M25. 
$a$ and $b$ are the smallest integers such that all automorphisms of $K[[x, y]]$ fixing both $\left(x, y^{a}\right)$ and $y$ and all automorphisms fixing both $x$ and $\left(x^{b}, y\right)$ also fix $I$. This is equivalent to saying that $a$ and $b$ are the smallest integers such that $I$ is expressible in terms of $\left(x, y^{a}\right)$ and $\left(x^{b}, y\right)$. In characteristic zero, this means $I$ is expressible as a polynomial in these two ideals. In positive characteristic, we can also use the Frobenius map. It was shown in [9] that the dimension of the orbit of $I$ is $a+b-2$. Thus, if $a=b=1$, then $I$ is a power of the ideal $(x, y)$, an image of such an ideal under a power of the Frobenius map, or the product of ideals of these types. These are the ideals that are invariant under all automorphisms of $K[[x, y]]$.

\section{Reducing the problem via fiber bundles}

If $I$ is a monomial ideal of finite colength in $K[[x, y]]$ with measuring sequence $\{a, b\}$, we can assume without loss of generality that either $a=b=1$ or $a>1$. The former case is very simple and has been discussed in the previous section. The latter problem can be made simpler through the introduction of a fiber bundle. If $I$ is not invariant under the involution switching $x$ and $y$, the automorphisms fixing $I$ also fix the ideal $\left(x, y^{2}\right)$. Thus the orbit of the pair of ideals $\left(I,\left(x, y^{2}\right)\right)$ is isomorphic to the orbit of $I$. Moreover the closure of the former orbit dominates the closure of the latter. In the case where $I$ is invariant under the involution of switching $x$ and $y$, the orbit of the pair of ideals $\left(I,\left(x, y^{2}\right)\right)$ is a double cover of the orbit of $I$. In both cases, the orbit of $\left(I,\left(x, y^{2}\right)\right)$ is a fiber bundle with respect to projection to the orbit of $\left(x, y^{2}\right)$. Moreover the closure is also a fiber bundle with respect to this projection. The fiber of the orbit is the orbit of $I$ under the stabilizer of $\left(x, y^{2}\right)$ and the fiber of the closure is the closure of this fiber. We let $F(I)$ denote the orbit of $I$ under the stabilizer of $\left(x, y^{2}\right)$ and $\bar{F}(I)$ its closure. To understand how $I$ degenerates, we need only understand the boundary of $\bar{F}(I)$.

\section{Ideal notation}

For the complicated ideals that come up in our examples, it will be convenient to use the following notation. Given a sequence of non-negative integers

$$
s=n_{1}, \ldots, n_{r}
$$

we will let $I(s)$ denote the ideal

$$
I(s)=\left(x^{r}, x^{r-1} y^{n_{1}}, \ldots, y^{n_{1}+\cdots+n_{r}}\right) .
$$

Although this notation for monomial ideals using sequences may seem cumbersome at first, in fact it makes the ideals easier to work with. For example for computing colengths we have

$$
\operatorname{col}\left(I\left(n_{1}, \ldots, n_{r}\right)\right)=\sum_{i=1}^{r}(r+1-i) n_{i} .
$$


Moreover, for multiplying ideals we have

$$
I\left(n_{1}, \ldots, n_{r}\right) I\left(m_{1}, \ldots, m_{k}\right)=I\left(l_{1}, \ldots, l_{r+k}\right)
$$

where

$$
l_{i}=\min _{\alpha+\beta=i}\left(\sum_{j=1}^{\alpha} n_{j}+\sum_{j=1}^{\beta} m_{j}\right) .
$$

We use these two formulas repeatedly in the last section.

\section{One-dimensional limits}

In this section we will work over the ring $R=K\left[\left[x_{1}, \ldots, x_{n}\right]\right]$. Let $I$ be a monomial of colength $d$ in $R$. Given a family of automorphisms $g(t)$ of $R$ parametrized by $K$, we get a map from $\mathbb{A}^{1}(K)$ into $\operatorname{Hilb}^{d}(R)$ sending $t$ to $g(t)(I)$. The closure of the graph in $\mathbb{P}^{1}(K) \times \operatorname{Hilb}^{d}(R)$ has a single point $(\infty, J)$ in the boundary. We say that $J$ is the flat limit of $g(t)(I)$ as $t$ goes to infinity. If $g(t)$ is of the special form mentioned in Theorem 5.1 below, we can find this limit by giving $R$ a grading preserved by $g(t)$ and then taking the limits of the homogeneous pieces of $I$.

The idea of this section is to take the limit $J$ of a family of automorphisms $g(t)$ applied to a monomial ideal $I$ in $R$ by putting weights on the variables of $R$ so that these automorphisms preserve the homogeneous pieces of $R$ with respect to these weights. Thus each homogeneous piece of $J$ is the limit of the correspond-

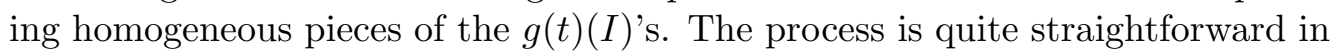
characteristic 0 , but slightly more subtle in positive characteristic.

We first develop some machinery for taking the limits of the homogeneous pieces of the images of $I$. We then apply our results to the space $\bar{F}(I)$ for $I$ of measuring sequence $\{3,1\}$ and $\{2,2\}$, determining the boundary ideal. In the next section we also apply these results to the measuring sequences $\{4,1\}$ or $\{3,2\}$.

Given a finite set of non-negative integers $T$ we define another set of integers $T^{\prime}$ of the same cardinality, which we call the $p$-shift of $T$, where $p$ is the characteristic in which we are working. If $p$ is 0 and the cardinality of $T$ is $n$, let $T^{\prime}$ be the set of integers from 0 to $n-1$. If $p$ is positive, we define $T^{\prime}$ by the following algorithm. Write all of the numbers in $T$ in base $p$. Let $n$ be the largest number of digits of any of these numbers. Add enough zeros at the beginning of each of the numbers in $T$ to make them all $n$ digits long in base $p$. Let $A=\left(a_{i, j}\right)$ be the matrix with each row corresponding to a number in $T$ and the $i^{\text {th }}$ column giving the $i^{\text {th }}$ digits of each of the numbers. Beginning with the first column and working to the right, we change each of the columns of $A$ as follows. Let $A_{i}$ be the matrix obtained after changing the first $i-1$ columns. Let $M_{i}$ be the matrix obtained by deleting the $i^{\text {th }}$ column of $A_{i}$. Let $A_{i+1}$ be the matrix with all columns except the $i^{\text {th }}$ the same as those of $A_{i}$ and the entry in the $j^{\text {th }}$ row and $i^{\text {th }}$ column giving the number of rows of $M_{i}$ above the $j^{\text {th }}$ row identical to the $j^{\text {th }}$ row. Thus, if all rows of $M_{i}$ are distinct, the $i^{\text {th }}$ column of $A_{i+1}$ will 
have all entries zero. In any case, all rows of $A_{i+1}$ are distinct. Let $T^{\prime}$ be the set of integers with base $p$ expansions given by the rows of $A_{n+1}$. For example, if $T=\{1,100\}$ and $p=3$ then $T^{\prime}=\{0,9\}$.

Lemma 5.1. Let $g(t)$ be the automorphism of $K[x]$ sending $x$ to $x+t$ for $t \in K$. Given a finite collection $T$ of non-negative integers, let $V(T)$ be the subspace of $K[x]$ generated by the polynomials $x^{e}$ for exponents $e \in T$. The limit of $g(t) V(T)$ as $t \in K$ goes to infinity is $V\left(T^{\prime}\right)$ where $T^{\prime}$ is the $p$-shift of $T$.

Proof. Let $T^{\prime \prime}$ be the set such that $V\left(T^{\prime \prime}\right)$ is the limit of $g(t) V(T)$ as $t \in K$ goes to infinity. If the characteristic of $K$ is zero, the lemma holds because $V\left(T^{\prime \prime}\right)$ must be invariant under $g(t)$. In the case of positive characteristic $p$, we proceed by induction on the sum of the elements of $T$. If the sum is zero, the lemma holds trivially. Suppose that there are subsets $T_{1}$ and $T_{2}$ of $T$ of cardinality one less than $T$ with $T_{1}^{\prime} \neq T_{2}^{\prime}$. Then $T_{1}^{\prime} \cup T_{2}^{\prime} \subset T^{\prime}$ and $V\left(T^{\prime \prime}\right)$ contains the limits of $g(t) V\left(T_{1}\right)$ and $g(t) V\left(T_{2}\right)$ as $t$ goes to infinity. By the inductive hypothesis, these are the sets $V\left(T_{1}^{\prime}\right)$ and $V\left(T_{2}^{\prime}\right)$. Thus $T^{\prime \prime}$ is forced to contain $T_{1}^{\prime}$ and $T_{2}^{\prime}$. If the union of $T_{1}^{\prime}$ and $T_{2}^{\prime}$ is $T^{\prime}$, we are reduced to the case in which there are no such subsets $T_{1}$ and $T_{2}$ of $T$.

Let $n$ be the largest number of digits of any element of $T$ base $p$. Let the matrices $A_{i}$ and $M_{i}$ be as in the definition above of $p$-shift above for our set $T$. Then the fact that we do not have subsets $T_{1}$ and $T_{2}$ with $T_{1}^{\prime} \neq T_{2}^{\prime}$ implies that all rows of $M_{i}$ occur exactly $m$ times for some integer $m$. Otherwise, the number of times some digit occurs as the first digit of an integer of $T_{1}^{\prime}$ after adding enough zeroes to the beginning of the integers to make them all $n$ digits, would depend on $T_{1}^{\prime}$. Thus the first digits of the integers in $T^{\prime}$ range from 0 to $m-1$. Since $V\left(T_{1}^{\prime}\right)$ is a subspace of $V\left(T^{\prime \prime}\right)$, the set $T^{\prime \prime}$ contains $T_{1}^{\prime}$ and hence differs from $T^{\prime}$ by at most one element $a$.

Let $f$ be the wedge product of the polynomials $(x+t)^{d}$ for $d \in T$. Then expanding $f$ into the sum of wedge products of monomials, the term with the highest coefficient of $t$ is the wedge product of basis elements of $V\left(T^{\prime \prime}\right)$. Let $f_{1}$ be the wedge products of the basis elements of $V\left(T_{1}\right)$. Then, since $T_{1}^{\prime} \subset T^{\prime \prime}$, this term is a multiple of the wedge product $x^{a} \wedge f_{1}$ where $a$ is the element of $T^{\prime \prime}$ not in $T_{1}^{\prime}$. Thus $a$ is the smallest number such that the coefficient of $x^{a} \wedge f_{1}$ in $f$ is non-zero, since the coefficient of this term will have the highest power of $t$.

For the rest of this proof, we will use bars to represent remainders modulo $x^{m p^{n-1}}$. So, for example $\overline{V(T)}$ is spanned by the elements $x^{d}$ for $d \in T$ less than $m p^{n-1}$. If $a$ has first digit less than $m$, the coefficient of $x^{a} \wedge f_{1}$ in $f$ is the same as its coefficient in $\bar{f}$. Moreover, if $\bar{f}$ is not identically zero, then the term with the highest power of $t$ is a multiple of $x^{a} \wedge f_{1}$. Thus the $V\left(T^{\prime \prime}\right)$ would be the limit as $t$ goes to infinity of $\overline{g(t) V(T)}$. We will now show that $\bar{f}$ is not zero. Let $S$ be the set of remainders of elements of $T$ modulo $p^{n-1}$. Then it is enough to show that for each $r \in S$ the remainders of the $m$ polynomials $\left(x^{p^{n-1}}+t^{p^{n-1}}\right)^{j}(x+t)^{r}$ modulo $x^{m p^{n-1}}$ for $j p^{n-1}+r \in T$ are independent. This follows from the fact the coefficients of the remainders of the polynomials $\left(x^{p^{n-1}}+t^{p^{n-1}}\right)^{j}$ can be 
put into an $m \times m$ matrix obtained from a Vandermonde matrix by elementary column operations not changing the determinant. Thus we see that $\bar{f}$ is not zero. Moreover, by a dimension count, we see that we can take the polynomials $x^{i p^{n-1}}(x+t)^{r}$ for $0 \leq i<m$ and $r \in S$ as a basis for $\overline{g(t) V(T)}$. Let $W_{i}$ be the span of these polynomials for a fixed $i$ with $r$ varying through $S$. Then

$$
\overline{g(t) V(T)}=\oplus_{i=1} W_{i}
$$

and the limit as $t$ goes to infinity of the direct sum of the $W_{i}$ 's is the direct sum of their limits. By our inductive hypothesis, the limit as $t$ goes to infinity of $W_{i}$ is $x^{i p^{n-1}} V\left(S^{\prime}\right)$, where $S^{\prime}$ is the $p$-shift of $S$. Hence, $T^{\prime \prime}$ is forced to be $T^{\prime}$ and the lemma follows.

Theorem 5.1. Let $I \subset R$ be a monomial ideal of finite colength. Let $h$ be a monomial not divisible by $x_{i}$. For each $t \in K$, let $g(t)$ be the element of $\operatorname{Aut}(R)$ with

for $j \neq i$ and

$$
g(t)\left(x_{j}\right)=x_{j}
$$

$$
g(t)\left(x_{i}\right)=x_{i}+t h .
$$

Let $J$ be the flat limit of ideals $g(t)(I)$. Then $J$ is characterized by the following. Let $x_{j}$ have weight $e_{j}$, where $e_{j}$ is the vector having $j^{\text {th }}$ coordinate 1 and all other coordinates 0 . Let $x_{i}$ have the same weight as $h$. Then each graded piece of $J$ is the limit of the corresponding graded piece of $I$. Let $M$ be a graded piece of $I$ and $T$ be the set of integers a such that a basis of $M$ is given by monomials of the form $x_{i}^{a} h^{d-a} f$ for $a \in T$ and $f$ a monomial of minimal degree. Then the corresponding graded piece of $J$ has a basis of monomials of the form $x_{i}^{a} h^{d-a} f$ for $a \in T^{\prime}$ where $T^{\prime}$ is the p-shift of $T_{M}$ described above.

Proof. Since $f$ and $h$ are invariant under $g(t)$, the proof reduces to Lemma 5.1.

Recall that $F(I)$ is the orbit of an ideal $I$ in $R$ under the automorphisms of $R$ fixing the ideal $\left(x, y^{2}\right)$ and $\bar{F}(I)$ is its closure. If $I$ has measuring sequence $\{3,1\}$ or $\{2,2\}$, then $F(I)$ is the $\mathbb{A}^{1}$ of ideals of the form $g(t)(I)$ where $g(t)$ is the automorphism $g(t) x=x+t y^{2}$ and $g(t) y=y$ or $g(t) x=x$ and $g(t) y=y+t x$ respectively. Hence the boundary point of $\bar{F}(I)$ is the limit of $g(t) I$ as $t$ goes to infinity.

Example: Consider the ideal $\left(x, y^{3}\right)^{n}$ for $n$ an arbitrary positive integer. On a case by case basis, one can use Theorem 5.1 to show that this ideal degenerates to $I(2,1,1)^{m}$ if $n=2 m$ and to $I(1,1) I(2,1,1)^{m}$ if $n=2 m+1$. These ideals are all integrally closed. To show this result for all $n$, one can use Proposition 7.1. We leave this as an exercise.

By comparing dimensions of graded pieces, one can see that $\left(x, y^{3}\right)^{n}+\left(y^{3 n-1}\right)$ degenerates to an ideal generated by a single monomial of the same weight as 
$y^{3 n-1}$ over the ideal that $\left(x, y^{3}\right)$. This can only be the monomial $x^{3 m+1}$ if $n=2 m+1$ and $x^{3 m-1} y$ if $n=2 m$. If $n$ is odd, this ideal is integrally closed. If $n$ is even, it is not integrally closed.

\section{Toric Varieties}

The following theorem will serve as the basis of our study of degenerations of monomial ideals in $K[[x, y]]$ with 3 -dimensional orbits.

Theorem 6.1. Let $I$ be a monomial ideal in $K[[x, y]]$ with finite co-length. If the orbit of $I$ under the automorphisms of $K[[x, y]]$ has dimension 3 , then either the normalization of the closure $\bar{F}(I)$ of the orbit of $I$ under the subgroup of those automorphisms stabilizing $\left(x, y^{2}\right)$ is a 2-dimensional toric variety, or the this holds after changing $I$ by exchanging $x$ and $y$.

Proof. Let $T$ be the 2-dimensional torus that acts by scaling $x$ and $y$. By [9], the ideal $I$ has measuring sequence $\{a, b\}$ where $a+b=3$. If $a$ is greater than 1 , then the action of $T$ on the orbit of $I$ restricts to an action on $F(I)$ since $\left(x, y^{2}\right)$ is invariant under $T$. Otherwise, exchanging $x$ and $y$ exchanges $a$ and $b$. The action of $T$ extends equivariantly to $\bar{F}(I)$.

If $I$ has measuring sequence $\{3,1\}$, then $F(I)$ is the $\mathbb{A}^{2}$ of ideals of the form $g(a, b)(I)$ where $g(a, b)(x)=x+a y^{2}+b y^{3}$ and $g(a, b)(y)=y$. If $I$ has measuring sequence $\{2,2\}$, then $F(I)$ is the $\mathbb{A}^{2}$ of ideals of the form $g(a, b)(x)=x+a y^{2}$ and $g(a, b)(y)=y+b x$. From the theory of toric varieties ([3] p. 61), we know that the boundary is a chain of rational curves, each separating limits of paths in $F(I)$ according to the limit of the ratio $a^{m}: b^{n}$ for some pair of integers $m$ and $n$. The intersections of these curves correspond to monomial ideals, since these are the $T$-invariant ideals. For each of these boundary curves, the fan corresponding to $\bar{F}(I)$ has a ray going through the corresponding point $(m, n)$. The other rays in the fan are the rays through $(-1,0)$ and $(0,-1)$ corresponding to the points in $F(I)$ with $b=0$ and $a=0$ respectively. We will use the notation $\Delta(I)$ to denote this fan. Moreover, we will let $I(m, n)$ denote the flat limit of $g(a, b)(I)$ for $a$ and $b$ functions of a parameter $t$ with $a^{m}$ asymptotic to a multiple of $b^{n}$. We will let $I^{+}(m, n)$ and $I^{-}(m, n)$ be the flat limits of $I(m, n)$ as the ratio $a^{m}: b^{n}$ tends to $1: 0$ and $0: 1$ respectively.

Next, we show how one can embed $F(I)$ in a larger projective space using $a$ and $b$ as coordinates in such a way that the closure of the image is $\bar{F}(I)$. Let $V$ be the quotient of the sum of all ideals corresponding to points in $F(I)$ by the intersection of all such ideals. Then $V$ is a finite dimensional vector space over $K$. The space $F(I)$ has an embedding in the Grassmannian of subspaces of $V$ of the appropriate dimension. This Grassmannian can in turn be embedded in projective space by Plücker coordinates. The coordinates of this projective space correspond to wedge products of monomials. By a homogeneity argument as in Lemma 8.1, the coordinate functions are monomials in $a$ and $b$. 
Next, we isolate the boundary of $\bar{F}(I)$. This boundary is the intersection of $\bar{F}(I)$ with the subspace of the ambient projective space characterized by its non-zero coordinates. Those non-zero coordinates can be found as follows. For each non-zero coordinate function $c a^{m} b^{n}$, plot the exponent vector $(m, n)$. The convex hull is a polytope with an edge perpendicular to each ray in $\Delta(I)$. The coordinate functions with exponent vector plotted on an edge are the only nonzero coordinates for the points in the curve corresponding to the perpendicular ray. The vertices of the convex hull correspond to the monomial ideals corresponding to points in $\bar{F}(I)$. For each of these, the corresponding point has only one non-zero coordinate, the one corresponding to the wedge product of monomials in the ideal.

Example: Let $I$ be the ideal $\left(x, y^{4}\right)$. Then $V$ is the quotient space $\left(x, y^{2}\right) /\left(x, y^{2}\right)^{2}$. The generators of $g(a, b)(I) /\left(x, y^{2}\right)^{2}$ as a vector space are $x+a y^{2}+b y^{3}$ and $x y+a y^{3}$. Taking the wedge product of these two generators we get $x \wedge x y+a x \wedge$ $y^{3}+a y^{2} \wedge x y+a^{2} y^{2} \wedge y^{3}+b y^{3} \wedge x y$. Plotting the exponent vectors of the coordinate functions, we see that the convex hull is a triangle. Thus, the boundary is a single irreducible curve corresponding to the edge through $(2,0)$ and $(0,1)$. Thus, this curve parametrizes ideals with wedge product of generators $a^{2} y^{2} \wedge y^{3}+b y^{3} \wedge x y$ $\bmod \left(x, y^{2}\right)^{2}$. These are the ideals of the form $\left(a^{2} y^{2}-b x y, x^{2}, x y^{2}, y^{3}\right)$. Using the notation $I(m, n), I^{+}(m, n)$, and $I^{-}(m, n)$ introduced earlier in this section, we have

$$
\begin{gathered}
\left(x, y^{4}\right)(2,1)=\left(a^{2} y^{2}-b x y, x^{2}, x y^{2}, y^{3}\right) \\
\left(x, y^{4}\right)^{+}(2,1)=\left(x^{2}, y^{2}\right)
\end{gathered}
$$

and

$$
\left(x, y^{4}\right)^{-}(2,1)=\left(x^{2}, x y, y^{3}\right)
$$

\section{Correspondences}

In the previous section, we saw how to find the orbits of certain ideals. However, we did not make use of the relations among such orbits. In this section we describe some correspondences that allow us to do this. The basic idea is to look at the orbit of a sequence of monomial ideals $\left(I_{1}, \ldots I_{n}\right)$ under the automorphisms of $K[[x, y]]$. As in the case of single ideals, we can assign a measuring sequence to the sequence of ideals. This measuring sequences determines this orbit up to a double cover ([9]). Moreover, adding the ideal $\left(x, y^{2}\right)$ into the sequence, the closure of the orbit of the sequence of ideals is a fiber bundle over the orbit of $\left(x, y^{2}\right)$. We will call the fiber $\bar{F}\left(I_{1}, \ldots, I_{n}\right)$. It is a correspondence in the product of the $\bar{F}\left(I_{j}\right)$ 's. This correspondence can be used to show that relations that hold among the $I_{j}$ 's continue to hold for their degenerations. In particular, we have the following proposition.

Proposition 7.1. Given ideals $I_{1}, I_{2}$, and $I_{3}$ with $I_{1} I_{2} \subset I_{3}$, for any point $P \in \bar{F}\left(I_{1}, I_{2}, I_{3}\right)$, we have $\pi_{1}(P) \pi_{2}(P) \subset \pi_{3}(P)$ where $\pi_{i}$ is projection to $\bar{F}\left(I_{i}\right)$. 
Proof. Let $V_{i}$ be the finite dimensional vector space obtained by taking the quotient of the sum of all ideals in $F\left(I_{i}\right)$ by the intersection of all ideals in $F\left(I_{i}\right)$. Consider the incidence correspondence

$$
\begin{gathered}
\Gamma=\left\{\left(\alpha_{1}, \alpha_{2}, \alpha_{3},\left(a_{1}, a_{2}, a_{3}\right)\right) \in V_{1} \times V_{2} \times V_{3} \times \bar{F}\left(I_{1}, I_{2}, I_{3}\right):\right. \\
\left.\alpha_{i} \in a_{i}, \alpha_{1} \alpha_{2}=\alpha_{3}\right\} .
\end{gathered}
$$

Then the set

$$
\left\{\left(\alpha_{1}, \alpha_{2}, \alpha_{3},\left(a_{1}, a_{2}, a_{3}\right)\right) \in V_{1} \times V_{2} \times V_{3} \times F\left(I_{1}, I_{2}, I_{3}\right): \alpha_{i} \in a_{i}\right\}
$$

is contained in $\Gamma$ and hence so is its closure

$$
\left\{\left(\alpha_{1}, \alpha_{2}, \alpha_{3},\left(a_{1}, a_{2}, a_{3}\right)\right) \in V_{1} \times V_{2} \times V_{3} \times \bar{F}\left(I_{1}, I_{2}, I_{3}\right): \alpha_{i} \in a_{i}\right\} .
$$

We will be using the above proposition as well as a few more below as our basic tools. This next proposition encapsulates the information we can obtain from Theorem 5.1.

Proposition 7.2. Given an ideal $I$ in $K[[x, y]]$ with measuring sequence $\{4,1\}$ or $\{3,2\}$, we define the automorphism $g(a, b)$ as in the beginning of the previous section (depending on the measuring sequence of I). Then, we have

$$
\begin{gathered}
I^{+}(0,-1)=I^{-}(1,0)=\lim _{t \rightarrow \infty} g(0, t)(I), \\
I^{-}(-1,0)=\lim _{t \rightarrow \infty} g(t, 0)(I), \\
I^{+}(1,0)=\lim _{t \rightarrow \infty} g(t, 0) I^{-}(1,0) .
\end{gathered}
$$

Moreover, if the characteristic of $K$ is not 2 and the measuring sequence of $I$ is $\{4,1\}$, then

$$
I^{-}(-1,0)=I^{+}(2,1)
$$

and

$$
I^{-}(2,1)=\lim _{t \rightarrow \infty} g(0, t) I^{+}(2,1)
$$

If the measuring sequence of $I$ is $\{3,2\}$, then in all characteristics

and

$$
I^{+}(1,0)=\lim _{t \rightarrow \infty} g(t, 0)(I)
$$

$$
I^{-}(0,1)=\lim _{t \rightarrow \infty} g(0, t) I^{+}(0,1) .
$$

Proof. One can verify by hand that

$$
\begin{gathered}
\left(x, y^{3}\right)^{+}(0,-1)=\left(x, y^{3}\right)^{-}(1,0)=\left(x, y^{3}\right), \\
\left(x, y^{4}\right)^{-}(-1,0)=\left(x, y^{4}\right)^{+}(2,1)=\left(x^{2}, y^{2}\right), \\
\left(x^{2}, y\right)^{-}(-1,0)=\left(x^{2}, y\right)^{+}(0,1)=\left(x^{2}, y\right) .
\end{gathered}
$$

Except for the ideal in the second line in characteristic 2 , these ideals are not invariant under the action of the automorphisms of $R$ fixing $\left(x, y^{2}\right)$. Thus the proposition follows. 
Proposition 7.3. Let $I$ be an ideal in $K[[x, y]]$ constructed from the ideals $\left(x, y^{4}\right)$ and $(x, y)$ (respectively $\left(x, y^{3}\right)$ and $\left.\left(x^{2}, y\right)\right)$. Consider $K[[x, y]]$ as a graded ring by letting $x$ have weight $n-m$ and $y$ have weight $2 n-3 m$ (respectively, letting $x$ have weight $n+m$ and $y$ have weight $n+2 m$ ). Then each graded piece of the monomial ideal $I^{+}(m, n)$ has the same dimension as the respective graded piece of the monomial ideal $I^{-}(m, n)$.

Proof. The ideal $I(m, n)$ is the direct sum of homogeneous pieces. Thus, the dimensions of each graded piece of the monomial ideals $I^{+}(m, n)$ and $I^{-}(m, n)$ must be the same as those of $I(m, n)$.

The idea of the next proposition is that while the exponent vector corresponding to a monomial ideal in $\bar{F}(I)$ may be some work to calculate, one can more easily calculate the vector going from two such exponent vectors corresponding to two ideals. From the relative position of the two exponent vectors, one may be able to argue that they are vertices joined by an edge in the convex hull of all the exponent vectors of coordinate functions embedding $F(I)$ as described earlier.

Proposition 7.4. Let $I$ be an ideal of measuring sequence $\{4,1\}$ or $\{3,2\}$. Given ideals $I_{1}=I^{+}\left(m_{1}, n_{1}\right)$ and $I_{2}=I^{+}\left(m_{2}, n_{2}\right)$ with

$$
\frac{m_{1}}{n_{1}}<\frac{m_{2}}{n_{2}}
$$

let $p_{1}$ be the product of the monomials in $I_{1}$ not in $I_{2}$ and let $p_{2}$ be the product of monomials in $I_{2}$ not in $I_{1}$. Let $\alpha$ and $\beta$ be the integers such that

$$
\frac{p_{1}}{p_{2}}=\frac{x^{\alpha}}{y^{\beta}} \text {. }
$$

If it is not possible to express

$$
\frac{2 \alpha-\beta}{3 \alpha-\beta}
$$

as the median of two distinct fractions strictly between

$$
\frac{m_{1}}{n_{1}} \text { and } \frac{m_{2}}{n_{2}}
$$

then $I_{1}=I^{-}(2 \alpha-\beta, 3 \alpha-\beta)$ and $I_{2}=I^{-}(2 \alpha-\beta, 3 \alpha-\beta)$.

Proof. The idea behind this proposition is that if the points $P_{1}$ and $P_{2}$ corresponding to $I_{1}$ and $I_{2}$ have certain relative positions in the polygon from which the fan $\Delta(I)$ is derived, then the convexity of the polygon forces the edge joining them to be an edge of the polygon. In particular, if the edge to the left of $P_{1}$ has left endpoint $P_{3}$ distinct from $P_{2}$, then by convexity, the slopes of the line segments joining $P_{3}$ to $P_{1}$ and $P_{2}$ must be between the slopes of the line segments of the edges of the polytope to the right of $P_{1}$ and to the left of $P_{2}$. This translates into the statement of the proposition. 
In the following theorem, we apply the techniques we have developed so far to determine the monomial ideals that the ideal $\left(x, y^{4}\right)^{n}$ can degenerate for any positive integer $n$. In addition, we give a conjecture describing all the ideals that $\left(x, y^{4}\right)^{n}$ can degenerate to. We also describe the obstruction to proving this conjecture.

Theorem 7.1. Let I denote the monomial ideal $\left(x, y^{4}\right)$ in $K[[x, y]]$. The monomial ideals that $I^{n}$ can degenerate to (up to exchanging $x$ and $y$ ) can be described as follows. Let $J\left(w_{1}, w_{2}, w\right)$ denote the monomial ideal generated by monomials $x^{c} y^{d}$ with $c w_{1}+c w_{2} \geq w$. Then up to permuting variables there are $[3(n+1) / 2]$ monomial ideals that $I^{n}$ can degenerate to, including itself. These are $I^{n},\left(x^{2}, y^{2}\right)^{n}$ and the ideals $J\left((r+1)^{2}, r^{2}, 2(r+1) r n\right)$ for $2 \leq r \leq n$ and $r=2 s$ for $2 \leq s \leq n$.

Proof. We will show that the rays in the fan $\Delta\left(I^{n}\right)$ are those through the points $(-1,0),(0,-1),(2,1),(4 m, 2 m-1)$ for $0 \leq m \leq n-1$ and $(2 m+1, m)$ for $3 \leq 2 m+3 \leq n$. The monomial ideals that $I^{n}$ can degenerate to are in bijection with the $[3(n+1) / 2]$ cones in between the rays of $\Delta\left(I^{n}\right)$ (up to exchanging $x$ and $y)$. Translating the ideals $J\left((r+1)^{2}, r^{2}, 2(r+1) r n\right)$ into sequence notation, it remains to prove the following.

1. $I^{n-}(-1,0)=I^{n+}(2,1)=\left(x^{2}, y^{2}\right)^{n}$.

2. $I^{n-}(2,1)=I(\underbrace{1, \ldots, 1}_{n}, 2, \underbrace{1, \ldots, 1}_{n-1})=I^{n+}(4 n-4,2 n-3)$.

3. Let $s=3,2,2,2$. Define the sequence $t_{n}$ corresponding to a boundary ideal of $F\left(I^{n}\right)$ inductively by letting $t_{0}$ be the empty sequence, $t_{1}=1,2$, $t_{2}=2,2,2$ and $t_{n}=t_{n-3} s$ for $n \geq 3$. Then $I^{n+}(0,-1)=I^{n-}(1,0)=I\left(t_{n}\right)$.

4. Let

$$
s_{0}=2, \underbrace{1, \ldots, 1}_{m-1}, 2, \underbrace{1, \ldots, 1}_{m}, 2, \underbrace{1, \ldots, 1}_{m}, 2, \underbrace{1, \ldots, 1}_{m}, 2,
$$

and

$$
s_{n}=\underbrace{1, \ldots, 1}_{m-n}, 2, \underbrace{1, \ldots, 1}_{m}, 2, \underbrace{1, \ldots, 1}_{m}, 2, \underbrace{1, \ldots, 1}_{m}, 2, \underbrace{1, \ldots, 1}_{i}
$$

for $1 \leq n \leq m$.

Define $t_{n}$ inductively by

$$
t_{n}= \begin{cases}\underbrace{\text { the empty sequence }}_{n}, \underbrace{1, \ldots, 1}_{n-1}, 2, \ldots, 1 & \text { for } n=0 \\
\underbrace{1, \ldots, 1}_{n-m-2}, 2, \underbrace{1, \ldots, 1}_{m}, 2, \underbrace{1, \ldots, 1}_{m}, 2, \underbrace{1, \ldots, 1}_{n-m-2} & \text { for } 1 \leq n \leq m+1 \\
t_{n-2 m-3} s_{j} & \text { for } m+2 \leq n \leq 2 m+2, \\
& \begin{array}{l}
\text { for } j+1 \equiv n \quad(\bmod m+1) \\
\text { and } n \geq 2 m+3 .
\end{array}\end{cases}
$$

Then $I^{n+}(4 m, 2 m-1)=I^{n-}(2 m+1, m)=I\left(t_{n}\right)$. 
5. Let

$$
\begin{gathered}
s_{n}= \begin{cases}2, \underbrace{1, \ldots, 1}_{m-1}, 2, \underbrace{1, \ldots, 1}_{m-1}, 2 & \text { for } n=0, \\
\underbrace{1, \ldots, 1}_{m-n}, 2, \underbrace{1, \ldots, 1}_{m-1}, 2, \underbrace{1, \ldots, 1}_{n} & \text { for } 1 \leq n \leq m, \\
\underbrace{1, \ldots, 1}_{2 m-n}, 2, \underbrace{1, \ldots, 1}_{m}, 2, \underbrace{1, \ldots, 1}_{n-m-1} & \text { for } m+1 \leq n \leq 2 m,\end{cases} \\
t_{n}= \begin{cases}\underbrace{1, \ldots, 1}_{n}, 2, \underbrace{1, \ldots, 1}_{n-1} & \text { for } 1 \leq n \leq m \\
t_{n-m-1}^{1, \ldots m p t y} s_{j} & \text { for } j+m+1 \equiv n \quad(\bmod 2 m+1) \\
& \text { and } n \geq m+1 .\end{cases}
\end{gathered}
$$

Then $I^{n+}(2 m-1, m-1)=I^{n-}(4 m, 2 m-1)=I\left(t_{n}\right)$.

Since $I^{+}(2,1)=\left(x^{2}, y^{2}\right)$, by Proposition $7.1, I^{n+}(2,1)=\left(x^{2}, y^{2}\right)^{n}$ for all $n$. By induction on $n$, for $n \geq 2$ the ideal $I^{n-}(2,1)$ contains

$$
I^{n-1+}(2,1) I^{+}(2,1)=I(\underbrace{1, \ldots, 1}_{n}, 2, \underbrace{1, \ldots, 1}_{n-2}, 2) .
$$

Proposition 7.3 shows that $I^{n-}(2,1)$ contains $y^{2 n+1}$. Checking colengths, this forces

$$
I^{n-}(2,1)=I(\underbrace{1, \ldots, 1}_{n}, 2, \underbrace{1, \ldots, 1}_{n-1}) .
$$

By Proposition 7.2 , we have $I^{2+}(0,-1)=I^{2+}(1,0)=I(2,2,2), I^{3+}(0,-1)=$ $I^{3-}(1,0)=I(3,2,2,2)$, and $I^{3+}(1,0)=I(1,2,2,2,1)$. By Proposition 7.1 it follows that $I^{n+}(0,-1), I^{n-}(1,0)$, and $I^{n+}(1,0)$ are as claimed.

We will verify the rest by induction on $n$. We have verified the theorem for $n=1$ in the example in the previous section. Supposing that $F\left(I^{k}\right)$ is as claimed for $k<n$ we will verify that $F\left(I^{n}\right)$ is as claimed. The fourth claim holds for all $m$, except possibly $m \leq n-2$ and $m=\frac{n-3}{2}$, by Proposition 7.1. For $n>2 m+3$ and $t_{i}$ as defined in (4) we have

$$
I\left(t_{n}\right)=I\left(t_{2 m+3}\right) I\left(t_{n-(2 m+3)}\right)+I\left(t_{m+1}\right) I\left(t_{n-(m+1)}\right) .
$$

For $m+2<n<2 m+3$ and $t_{i}$ as defined in (4) we have

$$
I\left(t_{n}\right)=I\left(t_{m+2}\right) I\left(t_{n-(m+2)}\right)+I\left(t_{m+1}\right) I\left(t_{n-(m+1)}\right) .
$$

The fifth claim holds for $m$ except $m \geq n-1$ and $m=\frac{n-1}{2}$ by Proposition 7.1. For $n>2 m+1$ and $t_{i}$ as defined in (5)

$$
I\left(t_{n}\right)=I\left(t_{2 m+1}\right) I\left(t_{n-(2 m+1)}\right)+I\left(t_{m+1}\right) I\left(t_{n-(m+1)}\right) .
$$

For $m+1<n<2 m+1$ and $t_{i}$ as defined in (5) we have

$$
I\left(t_{n}\right)=I\left(t_{m}\right) I\left(t_{n-m}\right)+I\left(t_{m+1}\right) I\left(t_{n-(m+1)}\right) .
$$


Using the inductive hypothesis on $n$, it remains to verify (4) for $3 \leq n \leq m+2$, (5) for $3 \leq n \leq m+1$, (4) for $n=2 m+3 \geq 5$ and (5) for $n=2 m+1 \geq 5$. We begin with the first two of these claims. We have already shown that

$$
I^{n-}(2,1)=I(\underbrace{1, \ldots, 1}_{n}, 2, \underbrace{1, \ldots, 1}_{n-1})
$$

and

$$
I^{n-}(4 n-8,2 n-5)=I(1,2, \underbrace{1, \ldots, 1}_{n-3}, 2, \underbrace{1, \ldots, 1}_{n-3}, 2,1) .
$$

It remains to find the monomial ideals in between these two ideals. By Proposition 7.1 these ideals contain the ideal

$$
I(1,2) I(\underbrace{1, \ldots, 1}_{n-1}, 2, \underbrace{1, \ldots, 1}_{n-2})=I(\underbrace{1, \ldots, 1}_{n}, 2, \underbrace{1, \ldots, 1}_{n-2}, 2) .
$$

This forces

$$
I^{+}(n,(2 n-5,4 n-8))=I(2, \underbrace{1, \ldots, 1}_{n-2}, 2, \underbrace{1, \ldots, 1}_{n-2}, 2) .
$$

By Proposition 7.4, there are no ideals between this ideal and the ideal $I^{n-}(2,1)$. Thus these two ideals are on the boundary divisor corresponding to the ray through the point $(4 n-4,2 n-3)$.

For $n \geq 5$, it remains to prove (4) for $n=2 m+3$ and (5) for $n=2 m+1$ assuming they hold for all smaller $n$. Let $r$ be an integer greater than 1 and let $n=2 r+1$. Given that

$$
\begin{aligned}
& I^{n-}(2(n-3), n-4)= \\
& \begin{cases}I(1,2,2,2,1,2,2,2) & \text { if } r=2 \\
I(1,2, \underbrace{1, \ldots, 1}_{r-2}, 2, \underbrace{1, \ldots, 1}_{r-2}, 2, \underbrace{1, \ldots, 1}_{r-2}, 2, \underbrace{1, \ldots, 1}_{r-1}, 2,1) & \text { if } r>2,\end{cases} \\
& I^{n+}(2(n-1), n-2)=I(\underbrace{1, \ldots, 1}_{r-1}, 2, \underbrace{1, \ldots, 1}_{r}, 2, \underbrace{1, \ldots, 1}_{r}, 2, \underbrace{1, \ldots, 1}_{r-1}),
\end{aligned}
$$

and that any ideal between these two must contain

$$
I(\underbrace{1, \ldots, 1}_{r}, 2, \underbrace{1, \ldots, 1}_{r-1}, 2, \underbrace{1, \ldots, 1}_{r-1}, 2, \underbrace{1, \ldots, 1}_{r-1})
$$

Proposition 7.3 forces

$$
I^{n-}(2(n-1), n-2)=I(\underbrace{1, \ldots, 1}_{r}, 2, \underbrace{1, \ldots, 1}_{r-1}, 2, \underbrace{1, \ldots, 1}_{r-1}, 2, \underbrace{1, \ldots, 1}_{r})
$$

and

$$
I^{+}(n,(n-4,2(n-3))=I(2, \underbrace{1, \ldots, 1}_{r-2}, 2, \underbrace{1, \ldots, 1}_{r-1}, 2, \underbrace{1, \ldots, 1}_{r-1}, 2, \underbrace{1, \ldots, 1}_{r-1}, 2) .
$$


Since there can be no ideals between these last two ideals, they both lie on the boundary divisor corresponding to the ray through the point $\left(n-2, \frac{n-3}{2}\right)$. By induction on $n$, we have proved the theorem.

The following conjecture describes the entire boundary of the space $\bar{F}\left(I^{n}\right)$.

Conjecture 7.1. Let I be the ideal $\left(x, y^{4}\right)$. Recall that the rays in the fan $\Delta\left(I^{n}\right)$ are those through the points $(-1,0),(0,-1),(2,1),(4 m, 2 m-1)$ for $0 \leq m \leq n-1$ and $(2 m+1, m)$ for $3 \leq 2 m+3 \leq n$. Given a ray through a point $(4 m, 2 m-1)$, let $f=b^{2 m-1} x^{2 m+1}+a^{4 m} y^{2 m+3}$. Then

$$
I^{n}(4 m, 2 m-1)=\sum_{i} f^{i} J(2 m+3,2 m+1,4(m+1)(n-(m+1) i)) .
$$

Given a ray through $(2 m+1, m)$ in $\Delta\left(I^{n}\right)$, let

$$
f=\left(b^{m} x^{m+1}+a^{2 m+1} y^{m+2} x\right)^{4} .
$$

Then

$$
I^{n}(2 m+1, m)=\sum_{i} f^{i} J(m+2, m+1,(2 m+3)(n-(2 m+3) i)) .
$$

Moreover

$$
I^{n}(2,1)=(x, y)^{2 n+3}\left(x^{2}, a^{2} y-b x y\right)^{n} .
$$

The difficulty in proving this conjecture is not in finding all of the ideals for a specific ray. This can always be done by Proposition 7.1. Rather, it is in finding the coefficients for the polynomial $f$ corresponding to each given ray. This involves taking determinants of large matrices. We describe these matrices in the next section.

\section{Matrices and lattice paths}

In the examples we have seen so far, the degenerations of the ideals have not depended on the characteristic of the field we were working in. However, this is something that we do not expect to happen in general. In this section, we consider some examples where the degenerations of ideals do depend on the characteristic. We will show that certain ideals can degenerate to others in exactly those characteristics not dividing the determinants of certain matrices. Considering the size and complexity of these matrices in the examples we will study, their determinants have relatively simple proven or conjectural formulas. These nice formulas all have interpretations in terms of the enumeration of lattice paths. Recall that a lattice path from the lattice point $(a, b)$ to the lattice point $(c, d)$ is a sequence of lattice points of minimal length starting at $(a, b)$ and ending at $(c, d)$ so that the distance between any two successive lattice points is 1. Unfortunately, a deeper reason for this connection to lattice paths remains elusive. Some of the matrices we will study will be binomial matrices, where the interpretation of the determinant in terms of the enumeration of lattice paths 
was given by Gessel and Viennot [4]. For other matrices, the relation to lattice paths comes from the fact that absolute values of the determinants form familiar sequences.

The following Theorem gives us a way of relating the characteristics in which a one dimensional limit of an ideal as in Theorem 5.1 has the same monomial generators as in characteristic zero to the enumeration of lattice paths. In particular, for monomial ideals with orbit of dimension 2 , the theorem determines the characteristics in which the monomial ideal in the boundary has the same generators as in characteristic 0 .

Theorem 8.1. Let $g(t)$ be an automorphism of $R$ with $g(t) y=y$ and $g(t) x=$ $x+$ th where $h$ is a monomial not divisible by $x$. Let $x$ have the same weight as $h$. Given a monomial ideal $I \subset R$, let $M$ be a graded piece of $I$ and $T$ be the set of integers a such that a basis of $M$ is given by monomials of the form $x^{a} h^{d-a} f$ for $a \in T$ and $f$ a monomial of minimal degree. Then the limit of $g(t) M$ has the same monomial generators in characteristic $p$ as in characteristic 0 if and only if for each graded piece $M, p$ does not divide the number of sets of non-intersecting lattice paths of cardinality $|T|$ such that the $i^{\text {th }}$ lattice path goes from $\left(0, m_{i}\right)$ to $(i-1, i-1)$, where the $m_{i}$ 's are the elements of $T$ in ascending order.

Proof. The $p$-shift of a set $T$ is the same as the 0 -shift of $T$ (i.e. $p$-shift in characteristic 0 ) if and only if the characteristic $p$ does not divide the divide the determinant of the binomial matrix

$$
\left(\left(\begin{array}{l}
a_{i} \\
b_{j}
\end{array}\right)\right)
$$

where the $a_{i}$ 's are the elements of $T$ in descending order and the $b_{i}$ 's are the elements of the 0 -shift of $T$ in descending order. This determinant is the number of $n$-tuples of non-intersecting lattice path such that the $i^{\text {th }}$ lattice path goes from $\left(0, a_{i}\right)$ to $\left(b_{i}, b_{i}\right)$.

The $p$-shift of $T$ is the same as the 0 -shift of $T$ in exactly those characteristics not dividing the coefficient of the wedge product of powers of $x$ with exponents in the 0 -shift of $T$ in the wedge product of powers of $(x+t)$ with exponents in $T$. This coefficient is the determinant of the binomial matrix above. The lattice path interpretation follows from Theorem 1 of [4]. The rest follows from Theorem 5.1.

In many cases these generators do not depend on the characteristic. For example, we have the following corollary.

Corollary 8.1. If $T$ is a set of consecutive integers, then the p-shift of $T$ is independent of the characteristic $p$. In particular, if $I$ is an ideal corresponding to a sequence $s$, then if $s$ consists of 2's 3's and 4's, the monomial generators of $I^{-}(1,0)$ are independent of characteristic. Moreover, if $s$ is a sequence of 3 's and 4 's, the monomial generators of $I^{-}(-1,0)$ are independent of characteristic. 
Proof. There is a unique $n$-tuple of non-intersecting lattice paths such that the $i^{\text {th }}$ one goes from $(0, i-1+k)$ to $(i-1, i-1)$ for a fixed positive integer $k$.

We now turn our attention to ideals corresponding to a sequence $s$ of the form

$$
s=\underbrace{4, \ldots, 4}_{n_{1}}, 3, \underbrace{4, \ldots, 4}_{n_{2}}
$$

and their degenerations. Let $I=I(s)$. From the corollary above, we know that

$$
I^{-}(0,-1)=I(\underbrace{1, \ldots, 1}_{2 n_{2}+2}, \underbrace{2,0, \ldots, 2,0}_{n_{1}})
$$

in all characteristics. By Proposition 7.2, in all characteristics except characteristic $2, I^{-}(2,1)$ can be found by taking a one-dimensional limit of $I^{-}(0,-1)$. This one-dimensional limit is $\mathfrak{m}^{n_{1}+2 n_{2}+2}\left(x^{n_{1}}, \mathfrak{m}^{n_{1}+1}\right)$ in all characteristics except 2 and those dividing the determinant of the $n_{1}$ by $n_{1}$ binomial matrix

$$
\left(\left(\begin{array}{c}
2\left(n_{1}+n_{2}+2-i\right) \\
n_{1}-2 i+j+1
\end{array}\right)\right) \text {. }
$$

By Theorem 26 (3.13) of [6], the determinant of this matrix is

$$
\left.2^{\left(n_{1}+1\right.}\right) f\left(n_{1}, n_{1}+n_{2}+1\right) / f\left(n_{1}, n_{1}\right)
$$

where

$$
f(r, n)=\prod_{i=0}^{\left[\frac{2 r-1}{4}\right]}\left(\begin{array}{c}
2(n-i) \\
2 r-4 i-1
\end{array}\right) .
$$

By Theorem 1 of [4], this is the number of $n_{1}$-tuples of non-intersecting lattice paths such that the $i^{t h}$ one goes from $\left(2 i-1,2 n_{1}+2 n_{2}+2+i\right)$ to $\left(n_{1}+i, n_{1}+i\right)$. This gives us a lattice path interpretation for the characteristics other than two in which $I$ can degenerate to $\mathfrak{m}^{n_{1}+2 n_{2}+2}\left(x^{n_{1}}, \mathfrak{m}^{n_{1}+1}\right)$. The following conjecture gives a lattice path interpretation in all characteristics.

Conjecture 8.1. Let I be the ideal

$$
I(\underbrace{4, \ldots, 4}_{n_{1}}, 3, \underbrace{4, \ldots, 4}_{n_{2}}) .
$$

Then I degenerates to $\mathfrak{m}^{n_{1}+2 n_{2}+2}\left(x^{n_{1}}, \mathfrak{m}^{n_{1}+1}\right)$ in exactly those characteristics not dividing the number of $n_{1}$-tuples of non-intersecting lattice paths such that the $i^{\text {th }}$ one goes from $\left(2 i-1, n_{1}+n_{2}+1+i\right)$ to $\left(n_{1}+i, n_{1}+i\right)$.

The number of lattice paths in the conjecture is the determinant of the $n_{1}$ by $n_{1}$ matrix

$$
\left(\left(\begin{array}{l}
n_{1}+n_{2}+2-i \\
n_{1}-2 i+j+1
\end{array}\right)\right)
$$

This follows from Theorem 2.7.1 of [11]. By Theorem 26 (3.13) of [6], the determinant of this matrix is $f\left(n_{1}, n_{1}+n_{2}+1\right) / f\left(n_{1}, n_{1}\right)$. 
We note that for $n_{1}$ equal to 2 or 3 , there is another lattice path interpretation for the determinants. Recall that one lattice path is said to dominate another if it lies above that lattice path and shares the same endpoints, but no other points. Let $K(p, q)$ be the sum of squares of numbers of lattice paths dominating each lattice path from the origin to $(p, q)$. It was shown in [8] that

$$
K(p, q)=\frac{(p+q+1) !(2 p+2 q+1) !}{(p+1) !(2 p+1) !(q+1) !(2 q+1) !} .
$$

For $n_{1}=2$ the determinant of the matrix above is $K\left(1, n_{2}+1\right)$ and for $n_{1}=3$ it is $K(2, k+1)$.

This conjecture can be verified (or shown to be false) for any individual value of $n_{1}$. We will verify it for $n_{1}$ equal to $1,2,3$, and 4 in Theorem 8.2. But, first we lay the necessary groundwork.

Recalling the method of finding the closure of $\bar{F}(I)$ by coordinate functions $c a^{m} b^{n}$, we observe that these coordinate functions are determinants of matrices with rows corresponding to monomials in $I$ and columns corresponding to monomials in the wedge product of monomials corresponding to the coordinate. The difficulty is not in finding the powers of $a$ and $b$ in the coordinate function, which can be determined by the lemma below, but in finding the coefficient $c$.

Example: Let $I$ be the ideal $\left(x, y^{4}\right)$. The monomials in $I$ not common to all ideals in $F(I)$ are $x$ and $x y$. The monomials in $\left(x^{2}, x y, y^{3}\right)$ not common to all ideals in $F(I)$ are $y^{3}$ and $x y$. Thus, the coordinate function for the embedding of $F(I)$ corresponding to the ideal $\left(x^{2}, x y, y^{3}\right)$ is the determinant of a $2 \times 2$ matrix with rows corresponding to $g(a, b)(x)$ and $g(a, b)(x y)$, columns corresponding to $y^{3}$ and $x y$, and entries giving the respective coefficients. This is the matrix

$$
\left[\begin{array}{ll}
b & 0 \\
a & 1
\end{array}\right]
$$

Without writing down any matrix, we could have have predicted that the determinant would be of the form $c b$ for some integer $c$ using the following lemma.

Lemma 8.1. Let $I$ be an ideal in $K[[x, y]]$ with measuring sequence $\{4,1\}$ (respectively $\{3,2\})$. Let $K[[x, y, a, b]]$ be a graded ring by giving $x$ weight $(1,0)$, $y$ weight $(0,1)$, a weight $(1,-2)$ and $b$ weight $(1,-3)$ (respectively $(-1,1))$. The product of monomials corresponding to the rows must have the same weights as the product of the monomials corresponding to the columns times the determinant.

Proof. The weights are chosen so that $g(a, b)$ sends monomials to homogeneous elements.

There seems to be no easy way to predict the coefficients of such matrices. This is the obstruction to proving the conjecture in the previous section. This coefficient can however be picked out by setting $a$ and $b$ equal to 1 in the matrix before taking the determinant. 
Notation: Given two monomial ideals $I$ and $J$ of the same colength in $K[[x, y]]$, let $\alpha(I, J)$ denote the coefficient of the coordinate function corresponding to $J$ for the embedding of $F(I)$. We specify the sign of $\alpha(I, J)$ as follows. Let $x$ have weight 2 and $y$ have weight one. Give monomials in $x$ and $y$ the ordering such that higher weights come before lower weights and such that among monomials of the same weight, those with higher power of $y$ come first. We make the convention that $\alpha(I, J)$ is the coefficient of the term having a wedge product of monomials in $J$ in descending order in the wedge product of $g(a, b)$ images of monomials in $I$ in descending order.

If $I$ corresponds to a sequence of 2's 3's and 4's and $J$ corresponds to a sequence of 1's and 2's, we can describe the matrix whose determinant gives $\alpha(I, J)$. Let $v(1)$ and $v(2)$ be the vectors giving the numbers of monic monomials in $I$ and $J$ of each weight, starting with the highest weight for which $I$ has any such ideals and going in descending order to the lowest such weight. Let $v(i, j)$ be the $j^{\text {th }}$ entry of $v(i)$. Let $v(3)$ be the vector of ascending positive integers with the number of $m$ 's being two less than the $m^{\text {th }}$ to last element in the sequence corresponding to $I$. Let $M^{k}\left(n_{1}, n_{2}\right)$ be the $n_{1}$ by $n_{2}$ matrix with entry $\left(\begin{array}{c}k \\ j-i\end{array}\right)$ in the $i^{\text {th }}$ row and $j^{\text {th }}$ column. Let $M(v(1), v(2), v(3))$ be the matrix made up of rows and columns of submatrices and the submatrix in the $i^{\text {th }}$ row and $j^{\text {th }}$ column of submatrices being

$$
\left(\begin{array}{c}
v(3, i) \\
j-i
\end{array}\right) M^{\left(\begin{array}{c}
v(3, i) \\
j-i
\end{array}\right)+i-j}(v(1, i), v(2, j))
$$

An example is given in Figure 1. The determinant of this matrix is $\alpha(I(4,4,4), I(1,1,1,2,1,1))$. From Theorem 7.1 , we know this has to be a unit.

Theorem 8.2. Conjecture 8.1 holds for $n_{1}$ from 0 to 4 .

Proof. Let $I_{n_{1}, n_{2}}$ be the ideal

$$
I(\underbrace{4, \ldots, 4}_{n_{1}}, 3, \underbrace{4, \ldots, 4}_{n_{2}})
$$

and $J_{n_{1}, n_{2}}$ be the ideal $\mathfrak{m}^{n_{1}+2 n_{2}+2}\left(x^{n_{1}}, \mathfrak{m}^{n_{1}+1}\right)$.

By Theorem 5.1 and Proposition 7.2 the ideal $I_{n_{1}, n_{2}}^{-}(2,1)$ is $J_{n_{1}, n_{2}}$ in characteristic zero. Therefore, $I_{n_{1}, n_{2}}$ degenerates to $J_{n_{1}, n_{2}}$ in exactly those characteristics not dividing $\alpha\left(I_{n_{1}, n_{2}}, J_{n_{1}, n_{2}}\right)$. The matrix having $\alpha\left(I_{n_{1}, n_{2}}, J_{n_{1}, n_{2}}\right)$ as its determinant, as described above, is a block lower triangular matrix with only the last block possibly having a nontrivial determinant. If $n_{1}=0$, all blocks are trivial. Otherwise, the last block is the matrix $M(v(1), v(2), v(3))$ where $v(1)$, $v(2)$ and $v(3)$ are the vectors of length $2 n_{1}$ given by

$$
\begin{gathered}
v(1)=2 n_{1}-1,2 n_{1}-1,2 n_{1}-3,2 n_{1}-3, \ldots, 3,3,1,1, \\
v(2)=2 n_{1}, 2 n_{1}-1, \ldots, n_{1}+2, n_{1}+1, n_{1}-1, n_{1}-2, \ldots, 1,0
\end{gathered}
$$

and

$$
v(3)=n_{2}+1, n_{2}+1, n_{2}+2, n_{2}+2, \ldots
$$




$\left[\begin{array}{llllll|lllll|llll|ll|l}1 & 1 & 0 & 0 & 0 & 0 & 0 & 0 & 0 & 0 & 0 & 0 & 0 & 0 & 0 & 0 & 0 & 0 \\ 0 & 1 & 1 & 0 & 0 & 0 & 0 & 0 & 0 & 0 & 0 & 0 & 0 & 0 & 0 & 0 & 0 & 0 \\ 0 & 0 & 1 & 1 & 0 & 0 & 0 & 0 & 0 & 0 & 0 & 0 & 0 & 0 & 0 & 0 & 0 & 0 \\ 0 & 0 & 0 & 1 & 1 & 0 & 0 & 0 & 0 & 0 & 0 & 0 & 0 & 0 & 0 & 0 & 0 & 0 \\ 0 & 0 & 0 & 0 & 1 & 1 & 0 & 0 & 0 & 0 & 0 & 0 & 0 & 0 & 0 & 0 & 0 & 0 \\ \hline 1 & 0 & 0 & 0 & 0 & 0 & 1 & 1 & 0 & 0 & 0 & 0 & 0 & 0 & 0 & 0 & 0 & 0 \\ 0 & 1 & 0 & 0 & 0 & 0 & 0 & 1 & 1 & 0 & 0 & 0 & 0 & 0 & 0 & 0 & 0 & 0 \\ 0 & 0 & 1 & 0 & 0 & 0 & 0 & 0 & 1 & 1 & 0 & 0 & 0 & 0 & 0 & 0 & 0 & 0 \\ 0 & 0 & 0 & 1 & 0 & 0 & 0 & 0 & 0 & 1 & 1 & 0 & 0 & 0 & 0 & 0 & 0 & 0 \\ 0 & 0 & 0 & 0 & 1 & 0 & 0 & 0 & 0 & 0 & 1 & 0 & 0 & 0 & 0 & 0 & 0 & 0 \\ \hline 1 & 0 & 0 & 0 & 0 & 0 & 2 & 2 & 0 & 0 & 0 & 1 & 2 & 1 & 0 & 0 & 0 & 0 \\ 0 & 1 & 0 & 0 & 0 & 0 & 0 & 2 & 2 & 0 & 0 & 0 & 1 & 2 & 1 & 0 & 0 & 0 \\ 0 & 0 & 1 & 0 & 0 & 0 & 0 & 0 & 2 & 2 & 0 & 0 & 0 & 1 & 2 & 0 & 0 & 0 \\ \hline 0 & 0 & 0 & 0 & 0 & 0 & 1 & 0 & 0 & 0 & 0 & 2 & 2 & 0 & 0 & 1 & 2 & 0 \\ 0 & 0 & 0 & 0 & 0 & 0 & 0 & 1 & 0 & 0 & 0 & 0 & 2 & 2 & 0 & 0 & 1 & 0 \\ 0 & 0 & 0 & 0 & 0 & 0 & 0 & 0 & 1 & 0 & 0 & 0 & 0 & 2 & 2 & 0 & 0 & 0 \\ \hline 0 & 0 & 0 & 0 & 0 & 0 & 1 & 0 & 0 & 0 & 0 & 3 & 3 & 0 & 0 & 3 & 6 & 1 \\ \hline 0 & 0 & 0 & 0 & 0 & 0 & 0 & 0 & 0 & 0 & 0 & 1 & 0 & 0 & 0 & 3 & 3 & 3\end{array}\right]$

Figure 1. $\mathrm{M}((5,5,3,3,1,1),(6,5,4,2,1,0),(1,1,2,2,3,3))$

The determinant of the matrix $M(v(1), v(2), v(3))$ is a polynomial in $n_{2}$ and hence it is enough to check finitely many values to verify the theorem for each value of $n_{1}$. We have checked these for $n_{1} \leq 4$.

\section{ACKNOWLEDGMENTS}

I would like to thank Jim Cogdell, Anthony Iarrobino, Steve Kleiman, Christian Krattenthaler, Monique Lejeune, David Wright, and Joachim Yameogo for helpful comments and discussion. I would also like to thank the referee for several helpful suggestions.

\section{References}

[1] V. I. Arnol'd, S. M. Gusein, A. N. Varchenko Singularities of Differentiable Maps Birkhauser, Boston, 1985-1988.

[2] J. Briançon, Description de Hilb ${ }^{n} \mathbb{C}\{x, y\}$. Inventiones math. 41 (1977), 45-89.

[3] W. Fulton, Introduction to Toric Varieties, Princeton University Press, Princeton, 1993.

[4] I. Gessel, G. Viennot Binomial Determinants, Paths, and Hook Length Formulae Advances in Mathematics 58 (1985), 300-321.

[5] A. Iarrobino, Punctual Hilbert schemes Mem. Amer. Math. Soc. 10 (1977).

[6] C. Krattenthaler, Advanced Determinant Calculus The Andrews Festschrift (Maratea, 1998). Sém. Lothar. Combin. 42 (1999).

[7] S. Kleiman, R. Piene Enumerating singular curves on surfaces, Contemp. Math., 241, Amer. Math. Soc, Providence, RI, 1999.

[8] G. Kreweras, H. Niederhausen Solution of an enumerative problem connected with lattice paths Europ. J. Combinatorics, 2 (1981), 55-60. 
schemes of points

[9] H. Russell, Alignment Correspondences (math.AG/0011213)

[10] Counting Singular Plane Curves Via Hilbert Schemes. Advances in Mathematics. 179 (2003), no. 1, 38-58.

[11] R. Stanley, Enumerative Combinatorics. Cambridge University Press 1 (1997).

[12] J. Yameogo, Décomposition cellulaire de variétés paramétrant des idéaux homogènes de $C \llbracket x, y \rrbracket$. Incidence des cellules. I. (French) Compositio Math. 90 (1994), 81-98.

Oklahoma State University, Department of Mathematics, 401 Mathematical SciENCES, Stillwater, OK 74078-8275

E-mail address: hrussell@mail.math.okstate.edu 